\title{
An Improved Model based on Viewer Response to Time- varying Video Quality for Video Telephony over LTE
}

\author{
Yao Sun, Fei Wang, Yuan Yao, Jing Wang, Zesong Fei \\ School of Information and Electronincs \\ Beijing Institute of Technology \\ Beijing, 100081, China \\ \{yao_sun, fei_wang, feizesong \}@bit.edu.cn
}

\begin{abstract}
The advent of LTE network's full deployment has led to a proliferation of mobile video services due to the greatly improved network conditions. One area of intense research is video telephony. Apparently operators are highly concerned about the quality of experience of the video telephony service perceived by end users. This paper proposes a method to evaluate and analyze LTE video telephony performance resorting to captured statistic information. Additionally, empirical results are used to derive analytical quality assessement models as a function of time, we introduce the effect of the viewer response to time- ${ }^{-}$arying video quality for video telephony into the existing models based on ITU-R G.1070. Through applying exponentially-weighted Minkowski summation, we could find that the correlation between the prediction of the improved model and subjective Mean Opinion Score becomes much better and the Pearson correlation coefficient could reach up to 0.96 through choosing appropriate parameters. Results have been validated on the basis of our own subjective video database in which video clips are reconstructed from captured files and subjective tests have been carried out involving 15 assessors using Single-Stimulus method in laboratory environment.
\end{abstract}

\section{CCS CONCEPTS}

- Networks $\rightarrow$ Network performance evaluation $\rightarrow$ Network performance modeling.

- Networks $\rightarrow$ Network performance evaluation $\rightarrow$ Network performance analysis.

\section{KEYWORDS}

*Produces the permission block, and copyright information $\dagger$ The full version of the author's guide is available as acmart.pdf document ${ }^{1}$ It is a datatype.

Permission to make digital or hard copies of all or part of this work for personal or classroom use is granted without fee provided that copies are not made or distributed for profit or commercial advantage and that copies bear this notice and the full citation on the first page. To copy otherwise, to republish, to post on servers or to redistribute to lists, requires prior specific permission and/or a fee. Mobimedia 2017, July 13-14, Chongqing, People's Republic of China Copyright $\odot 2017$ EAI 978-1-63190-156-0
LTE, Video telephony, QoE, Exponentially-weighted Minkowski summation, Recency effect.

\section{ACM Reference format:}

Yao Sun, Fei Wang, Yuan Yao, Jing Wang and Zesong Fei. 2017. SIG Proceedings Paper in word Format. In Proceedings of ACM MobiMedia conference, Chongqing, China, fuly 2017 (MOBIMEDIA'17), 7 pages. DOI: $10.1145 / 1234$

\section{INTRODUCTION}

In recent years, commercial LTE has been fully deployed. A wider bandwidth IP network has been established which enables video telephony service over LTE network. Obviously, operators pay close attention to the quality of experience perceived by users for the service they provide. Therefore, assessing the video quality of video telephony service over LTE network is essential. The video quality assessment methods could be classified into subjective methods and objective methods [1]. Subjective methods are viewed as the most accurate ways to measure quality of experience of end users because they test the experience of users in a direct way by enquiring assessors to obtain the subjective Mean Opinion Score(MOS). But they suffer from certain defects, such as time consuming, human resources consuming, unrepeatable. Consequently, developing an objective video quality assessment method is indispensable for operators to do QoE monitoring and network planning.

Among objective models, no reference models focus on mapping the network statistics and media statistics to the quality assessment which is compliant with the network services like video telephony. Due to a lack of well-developed standard methods to get these information, this paper presents a concrete way to capture the data packets during video callings between two Android smart phones over LTE. We can extract statistic information from these captured packets to analyze and evaluate the video telephony performance over LTE. On the grounds of the analysis results, we develop improvements to a kind of existing video quality assessment model for video telephony application by including the viewer response to time-varying video quality.

The rest of this paper is organized as follows: Section 2 presents a brief description of video telephony over LTE, QoE and related works. In Section 3, we introduce a test method to obtain 
the statistic information of the video telephony and gives an analysis. Section 4 provides the improved video quality assessment model and analyses its performance. Conclusions are drawn in Section 5.

\section{Background and related works}

\subsection{Video telephony over LTE}

Voice over Long-Term Evolution (VoLTE) is widely known as a voice calling solution for next generation network. Based on IP Multimedia Subsystem (IMS) network, it supports high-speed wireless communication for mobile phones and data terminals. Furthermore, it supports video calling which is also known as video telephony over LTE. Video telephony over LTE uses the same control plane protocol as VoLTE which is called the Session Initiation Protocol (SIP). The GSMA profile for IMS conversational video [3] applies to video telephony over LTE adding video capability and providing end users with synchronized fullduplex voice and video streams. The well established H.264 codec is implemented to encode and decode video streams in video telephony over LTE. It delivers superior quality running Video Graphics Array (VGA) resolution.

\section{$2.2 \mathrm{QoE}$}

QoE (Quality of Experience) is a kind of measurement of a user's experiences with a specific service. It evaluates service quality from the users' perspective. QoE in the context of telecommunications networks is defined as: The degree of delight or annoyance of the user of an application or service [4]. It results from the fulfillment of his or her expectations with respect to the utility and / or enjoyment of the application or service in the light of the user's personality and current state. QoE is related to QoS (Quality of Service), so we could call QoE is user-level QoS [5]

\subsection{Existing models}

There have been significant research efforts in the domain of video telephony applications. ITU-T Recommendation G.1070 [7] describes a computational model for video quality estimation for video telephony applications over IP network. The video quality estimation $V_{q}$ is calculated as shown in Equation (1):

$$
V_{q}=1+I_{c} e^{\frac{P_{p l v}}{D_{P p l v}}}
$$

where $I_{C}$ represents the basic video quality, determined by codec distortion and it is a function of the bit rate and frame rate $P_{p l v}$ is the packet loss rate and $D_{P p l v}$ presents the degree of video quality robustness against packet loss, which is dependent on codec type, video format, video display size and key frame interval. The basic video quality $I_{C}$ is expressed in Equation (2):

$$
I_{c}=v_{3}\left(1-\frac{1}{1+\left(\frac{b}{v_{4}}\right)^{v_{5}}}\right)
$$

where $b$ is the bit rate and $v_{3}, v_{4}, v_{5}$ are the coefficients which are dependent on codec type, video display size, key frame interval and must be calculated using subjective video quality tests. Provisional values are provided only for MPEG-4 in QVGA and QQVGA video formats.

Based on ITU-R G.1070 model, the influences of video content are taken into account in [8]. It can be seen that video quality strongly depends on video contents. According to a subjective estimation for the clip movements, this paper classifies the clip movements into 3 groups: "Low", "Medium" and "High". They propose to use 3 different sets of values for $v_{4}$ and $v_{5}$ depending on the movement contents. 3 different sets of values proposed are shown in the following table:

Table 1: $v_{4}, v_{5}$ values for each group for MPEG-2

\begin{tabular}{llcc}
\hline Movement & $v_{3}$ & $v_{4}$ & $v_{5}$ \\
\hline Low Movement & 4 & 0.366 & 1.32 \\
Middle Movement & 4 & 0.67 & 1.36 \\
High Movement & 4 & 1.088 & 1.56 \\
\hline
\end{tabular}

$$
V_{q}=1+4 k\left(1-\frac{1}{1+\left(\frac{a b}{v_{4}}\right)^{v_{5}}}\right)
$$

The model is shown in Equation (3), where $k$ is called enhancement factor, depending on codec, display size and bit rate and $a$ is bit rate scale factor depending on display size.

In [9], the same model is presented in equation with including packet-loss degradation characteristics and they calculated the coefficients table for H.264 coding format. The model can be seen as follows:

$$
V_{q}=1+4 k\left(1-\frac{1}{1+\left(\frac{a b}{v_{4}}\right)^{v_{5}}}\right) e^{\frac{-P L F}{v_{p}}}
$$

\section{LTE Video telephony performance analysis and results}

In order to explore the LTE video telephony performance, a test system under real LTE network has been set to conduct the whole research. Specific information about our network environment is presented in Table 2.

We proceed LTE video calls by using two Android cellphones. Each terminal device is held by one test personnel. During the call, we would go on with capturing RTP packets which video calls use to achieve data transmission. Each cellphone is controlled by a laptop named control terminal, which sends commands to the cellphone to control the capturing flow. Captured files are finally uploaded to management and analysis terminal. 
A QoE Model based on Viewer Response to Time-varying Video Quality for Video Telephony over LTE

Table 2: Specific information about LTE network.

\begin{tabular}{lc}
\hline Parameters & values \\
\hline Network operator & China mobile \\
Network type & LTE \\
Duplex mode & TDD \\
Frequency band & B40: $2320 \mathrm{MHz}-2370 \mathrm{MHz}$ \\
& B39: $1880 \mathrm{MHz}-1900 \mathrm{MHz}$ \\
& B41: $2575 \mathrm{MHz}-2635 \mathrm{MHz}$ \\
\hline
\end{tabular}

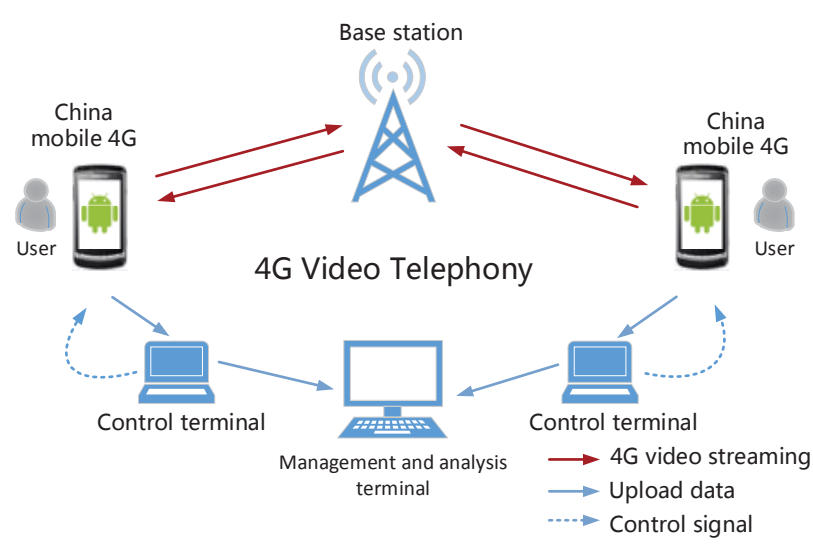

Figure 1: The test system framework.

As depicted in Figure 1, the test system framework is composed of 3 parts: management and analysis terminal, control terminals and end devices (cellphones). Control terminals are computers on which running the adb tools to manipulate the whole capturing flow. Cellphones are connected with the corresponding control terminals through USB cable. Tcpdump [10] application is embedded in the Android cellphone as the capturing tool. Tcpdump is a widely used command-line packet analyzer that works on most Unix-like operating systems: Linux, Solaris, BSD, macOS, Android and so on and it's free. Tcpdump uses the libpcap library to capture packets and displays packets being transmitted or received over a network.

Once video call is established, data packets will be captured and saved in a ".pcap" file. Then we transfer this file to the management and analysis terminal which is also a computer running Wireshark [11]. Wireshark is the world's foremost network protocol analyzer which allows you to see what's happenning or what happened on your network at a microscopic level and it's open source. Details about system components are presented in Table 3.

Aiming to accomplish the following research, we have to limit ourselves to a certain number of test cases. We excogitate 4 specific cases in which the influnce of different environmental conditions combinations on QoE could be well studied. Details of the test case settings are given in Table 4 .
MobiMedia'17, July 2017, Chongqing, China

Table 3: Details about system components.

\begin{tabular}{lcc}
\hline Components & Amount & Configuration \\
\hline End devices & 2 & $\begin{array}{c}\text { Xiaomi 5 standard edition, } \\
\text { Android 6.0.1, } \\
\text { baseband version: TH20c1.9- }\end{array}$ \\
& & 00038 \\
Control terminal & 2 & Windows 7 ultimate \\
& & CPU: Intel(R) Core i5-5200U \\
Memory: 4GB \\
$\begin{array}{l}\text { Management and } \\
\text { analysis termial }\end{array}$ & 1 & Windows 7 ultimate \\
& & CPU: Intel(R) Core i5-4590 \\
Memory: 8GB
\end{tabular}

Table 4: Test case settings.

\begin{tabular}{lcccc}
\hline $\begin{array}{l}\text { Test } \\
\text { case }\end{array}$ & Environment & Movement & $\begin{array}{c}\text { Number } \\
\text { of times }\end{array}$ & $\begin{array}{c}\text { Duration } \\
\text { at a time }\end{array}$ \\
\hline Case 1 & Indoor & Low & 50 & $13-30 \mathrm{~s}$ \\
Case 2 & Indoor & Middle/high & 50 & $13-30 \mathrm{~s}$ \\
Case 3 & Outdoor & Low & 20 & $13-30 \mathrm{~s}$ \\
Case 4 & Outdoor & Middle/high & 20 & $13-30 \mathrm{~s}$ \\
\hline
\end{tabular}

On the whole, our test method is conducted under real LTE network which is different from those under simulation network environment. That makes it more valuable in future work planning and guiding significance. And real-time capturing is indispensable for information collection, which reflects the situation of LTE network more factually. Another important part of our method is that we reconstructed the original videos and reduced videos of video calls in pairs by using a Wireshark plugin. We could obtain media statistic information such as bitrates, framerate and resolution and these videos are also used to do subjective tests. So it's of great importance to retain primitiveness. In our method, these video are totally lossless comparing to the most primitive display during video calls unlike other screen recording methods which would bring secondary damage.

We conduct 50 video calls for each case in indoor environment and 20 video calls for each case in outdoor environment. The duration of video call at a time ranges from 13 seconds to 30 seconds. After doing all these test cases, network statistic information and media statistic information are collected and captured files are saved on local host. With these reconstructed videos, the packet loss rates and other network statistic information can also be calculated by using Wireshark. The details of the average statistic information of all video calls are gathered in Table 5.

Seen in this table, bit rates and packet loss rates differ greatly in different environments. In outdoor environment, we could observe that owing to a favorable network condition, there are few packets that are lost during video calls and the bit rates stabilize around 650kbps. Nevertheless, high packet loss rates took place in indoor environment because of terrible network condition and the bit rates are apparently lower than those in outdoor environment. 
Table 5: The average statistic information

\begin{tabular}{lcccc}
\hline $\begin{array}{l}\text { Test } \\
\text { case }\end{array}$ & $\begin{array}{c}\text { Packet } \\
\text { loss rate }\end{array}$ & $\begin{array}{c}\text { Bitrate } \\
(\mathrm{kbps})\end{array}$ & $\begin{array}{c}\text { Framerate } \\
(\mathrm{fps})\end{array}$ & Resolution \\
\hline Case 1 & $17.9654 \%$ & 418.68 & 25 & $640 \times 480$ \\
Case 2 & $18.5632 \%$ & 422.02 & 25 & $640 \times 480$ \\
Case 3 & $0 \%$ & 656.50 & 25 & $640 \times 480$ \\
Case 4 & $0.408 \%$ & 642.30 & 25 & $640 \times 480$ \\
\hline
\end{tabular}

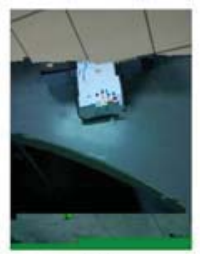

(a) $1 \mathrm{~s}$

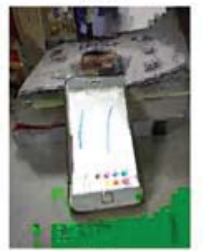

(e) $5 \mathrm{~s}$

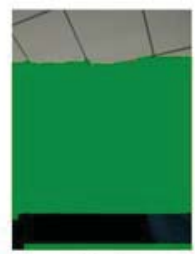

(b) $2 \mathrm{~s}$

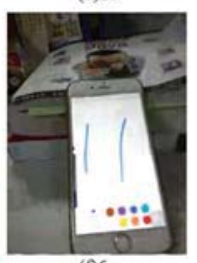

(f) $6 \mathrm{~s}$

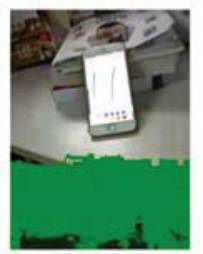

(c)3s

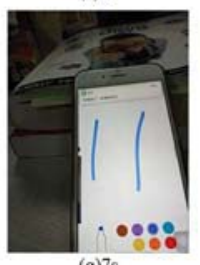

(g) $7 \mathrm{~s}$

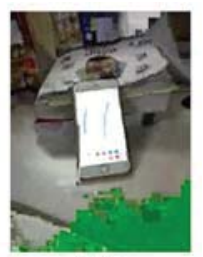

(d) $4 \mathrm{~s}$

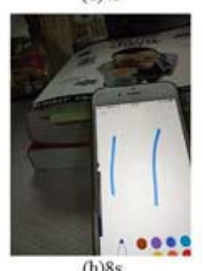

Figure 2: A screenshot each second at the beginning of a video call.

On the basis of our investigation on captured files, another notable fact is that in the precious first several seconds of a video call in indoor environment, the video quality is really bad with severe blur and blockiness. We made a screenshot each second at the beginning of a video call seen in Figure 2 .

We could see that when the time moves forward during a video call, the video quality is generally getting worse and worse. Another research has been made on the packet loss rates and bitrates during the whole session of a video call.

Figure 3 reveals the fluctuation of the bitrate and packet loss rate in one video call session. Clearly seen in Figure 3, the bitrate is relatively stable as compared to the packet loss rate within the range of $400 \mathrm{Kbps}$ to $700 \mathrm{Kbps}$. But the packet loss rate has a huge fluctuation in the beginning of the video call and its peak value could come up to $60 \%$. After that, the packet loss rate goes back to a steady state in which packet loss barely happens. In this respect, for a video call, packet loss rate is the key factor that affects the video quality.

Furthermore, we calculate the average packet loss rates of 100 video clips in indoor environment in each second at the first 10 seconds of video calls as shown in Figure 4.

Obviously, for 100 video clips ( 50 with low movement and 50 with middle (high movement in indoor environment), at the first second of video calls, the average packet loss rate could reach up to $45 \%$, then the packet loss rate drops rapidly to $5 \%$ below at about

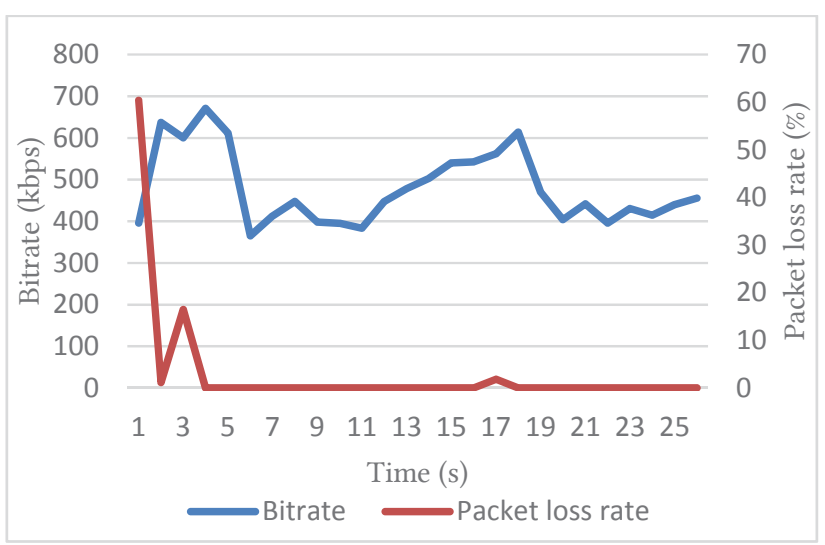

Figure 3: Packet loss rate and bitrate of a session.

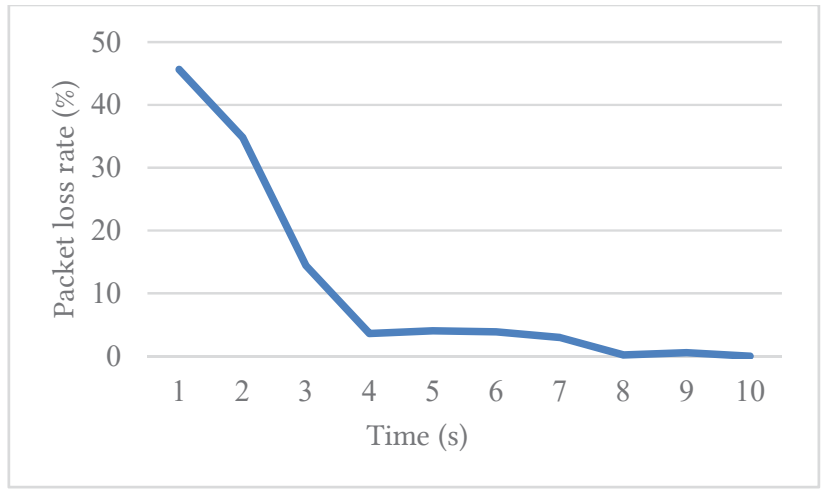

Figure 4: Average packet loss rate at the first 10 seconds of video calls.

the fourth second. And after 8 seconds during the call, packet loss rarely occurs.

So in a more general way, we confirm that the main concentration of packet loss is in the beginning of video calls. So a high average packet loss rate of a video call does not mean a continuous terrible experience during the whole process of calls. We made a further exploration about why the packet loss rate is excessively high at the beginning of video calls.

Figure 5 shows the IO graphs of video calls. Each wave form represents a video call and there are 10 video calls in total shown in this graph. As we could see, for each video call, the number of exchanged packets reached its peak value at the very beginning, which may result from network congestion with the limitation of bandwidth.

Since a standard database of test LTE video telephony videos is not available yet, we have generated a video telephony video database which is composed of reconstructed original videos and reconstructed reduced videos as discussed in aforementioned part. There are 19 video telephony clips in our database, 8 for low movement, 6 for middle movement and 5 for high movement. And their packet loss rates range from $0.1 \%$ to $40 \%$. 


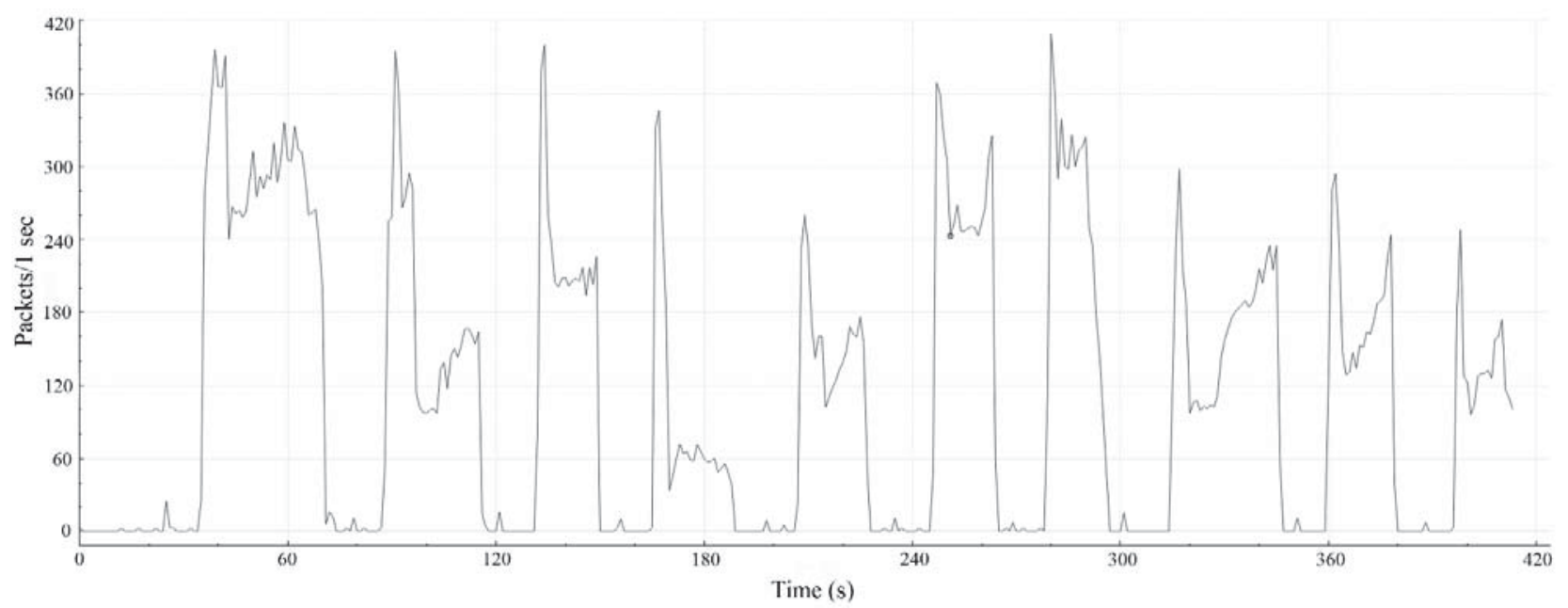

Figure 5: Video telephony over LTE IO graphs.

Table 6: The detail of the video telephony database.

\begin{tabular}{lc}
\hline Parameters & Values \\
\hline Number of sequences & 19 \\
duration of each video & ranges from $13 \mathrm{~s}$ to $30 \mathrm{~s}$ \\
packet loss rate of each video & ranges from $0.1 \%$ to $40 \%$ \\
framerate & $25 \mathrm{fps}$ \\
bitrate & ranges from $400 \mathrm{kbps}$ to \\
coding format & $700 \mathrm{kbps}$ \\
\hline
\end{tabular}

The detail of the video database is shown in Table 6.

We conducted subjective experiments basing on our video database by using Single-Stimulus Method in a laboratory environment. Fifteen subjects who are students in Beijing Institute of Technology aged 20-25 participated in this experiment. They are nonexperts who are not directly concerned with multimedia quality as part of their work.

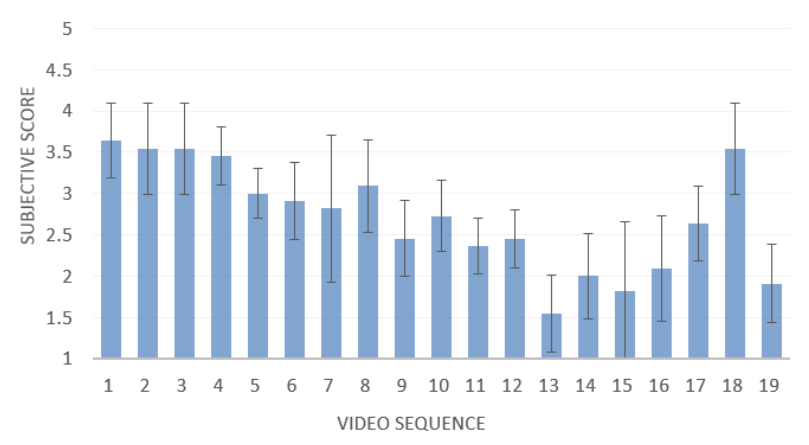

Figure 6: MOS (with confidence interval) of each video clips in the database.
The video quality scores obtained from the subjective test are summarized in Figure 6.

\section{Improved model and experimental results}

For the original model represented in equation(3), bitrate and packet loss rate are the most two significant factors that impacts the video quality. Here now we assume that in a specific situation the movement in video clips is low, the resolution is VGA and the coding format is H.264. We got the following two figures showing original model prediction as a function of the bitrate (in Figure 7 , supposing packet loss rate is a constant) and the packet loss rate (in Figure 8, supposing bitrate is a constant) respectively.

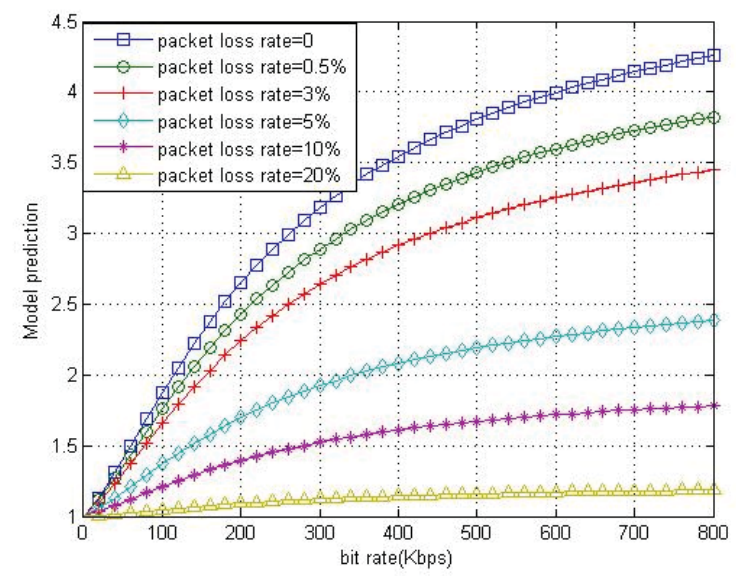

Figure 7: Model prediction as a function of the bitrate for 6 different packet loss rates.

Figure 7 shows the relation between model prediction and the bitrates. As can be seen, for video telephony as mentioned above, bitrates center on the range of $400 \mathrm{Kbps}$ to $700 \mathrm{Kbps}$, which 
indicates a small change in subjective scores with having packet loss. Specifically MOS changes are less than 0.5. Whereas a relative high packet loss rate, which probably occurs in video telephony, has a big impact on subjective quality of experience regardless of bitrates' flunctuation as demonstrated in Figure 8. Specifically MOS changes are more than 1.

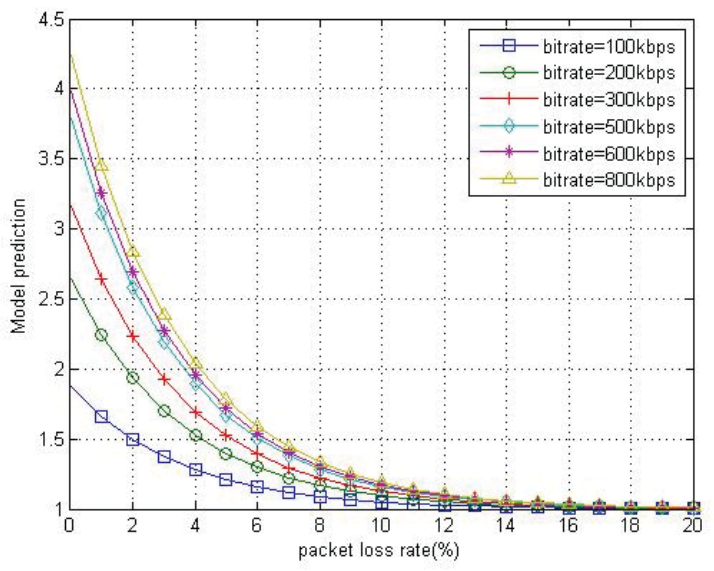

Figure 8: Model prediction as a function of the packet loss rate for 6 bitrates.

The prediction enhancement of the objective model is measured with the Pearson linear correlation coefficient, given by:

$$
\rho_{X, Y}=\frac{N \sum X Y-\sum X \sum Y}{\sqrt{N \sum X^{2}-\left(\sum X\right)^{2}} \sqrt{N \sum Y^{2}-\left(\sum Y\right)^{2}}}
$$

Subjective MOS results are used as $Y$ and scores from the objective models are used as $X$, and $N$ is the length of data $\operatorname{sets}(X$ or $Y$ )

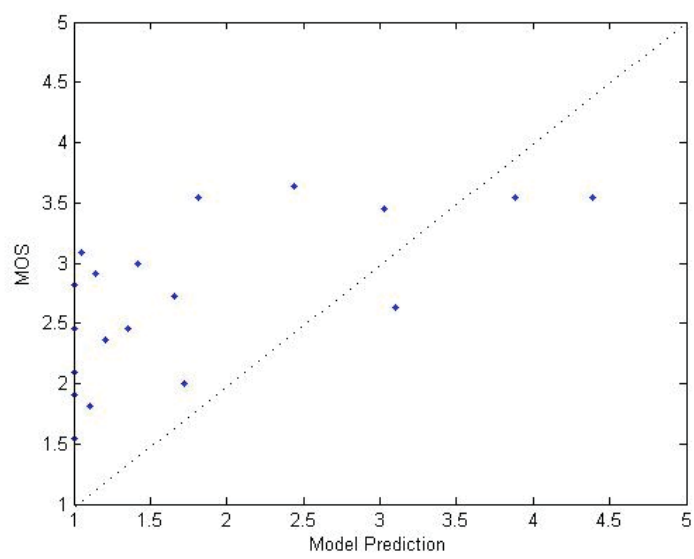

Figure 9: Scatter plots of MOS versus scores calculated from original model.
Based on original existing model formulated as Equation (3), described in [8] and [9], we calculated the predicted scores for 19 video clips in our subjective video database. The correlation results are presented in Figure 9.

As the results show, the correlation between subjective MOS and scores from the original model is very bad in low scores scale. The Pearson correlation coefficient is around 0.64 . Some video clips got 3 in subjective scores but only got 1 in original model prediction probably because of high packet loss rate in a video call session. Thus, we could find limitations of the original model.

From the previous analysis, bitrates and packet loss rates during a video call change as time goes on. Thus, the video quality varies as a function of time, particularly reaching a negative-peak at the beginning of video calls. So a temporal pooling method is imperative for video quality assessment of video telephony.

We further consider the impact of time-varying video quality on users' quality of experience, particularly influenced by what they see in the last part of the video clips, namely, recency effect. In other words, assessors attach more weight to artifacts appearing in the end of the sequence. If the end of sequence is of great quality, assessors may upgrade the final rating and vice versa. Another important fact is that overall ratings are greatly affected by the peak intensity of the clip. It seems that human subjects give disproportionate weight to those moments that have extreme quality, good or bad. So we introduce a temporal pooling method called exponentially-weighted Minkowski summation, which includes the response to these two effects into the existing model. This pooling method was formulated according to the following expression:

$$
O M_{\text {expMink }}=\left[\frac{1}{T} \sum_{t=1}^{T} \exp \left(\frac{t-T}{\operatorname{tau}}\right) O M^{p}(t)\right]^{\frac{1}{p}}
$$

where tau is the exponential time constant controlling the strength of the recency effect and high values of tau is corresponding to a higher recency effect. Parameter $p$ is given a meaning of Minkowski exponent. Values of $p$ in the range 1-12 were examined and high values of $p$ emphasize the influence by the highest scores on the overall sequence. $T$ is the duration of the video clip. $O M$ stands for objective models, to be specific, for video telephony as mentioned in section 2 , showing as follows:.

$$
V_{q}=1+4 k\left(1-\frac{1}{1+\left(\frac{a b}{v_{4}}\right)^{v_{5}}}\right) e^{\frac{-P L F}{v_{p}}}
$$

So through employing exponentially-weighted Minkowski summation, $V q$ becomes a function of time and bit rate and packet loss rate also turn into being time-varying. We calculated these two parameters in each second. Thus, for one video clip, we may get multiple scores calculated from original objective model in a time-based order. At the end, these scores make one integrated score by applying temporal scheme. And the model becomes the following one: 
A QoE Model based on Viewer Response to Time-varying Video Quality for Video Telephony over LTE

$$
\begin{aligned}
V_{q}=\left[\frac{1}{T} \sum_{t=1}^{T} e^{\frac{t-T}{\operatorname{tau}}(} 1\right. & +4 k(t) \\
& \left.\left.\cdot\left(1-\frac{1}{1+\left(\frac{a b(t)}{v_{4}}\right)^{v_{5}}}\right) e^{\frac{-P L F(t)}{v_{p}}}\right)\right]^{\frac{1}{p}}
\end{aligned}
$$

The correlation results obtained for the improved model with introducing exponentially-weighted Minkowski summation are plotted in Figure 10.

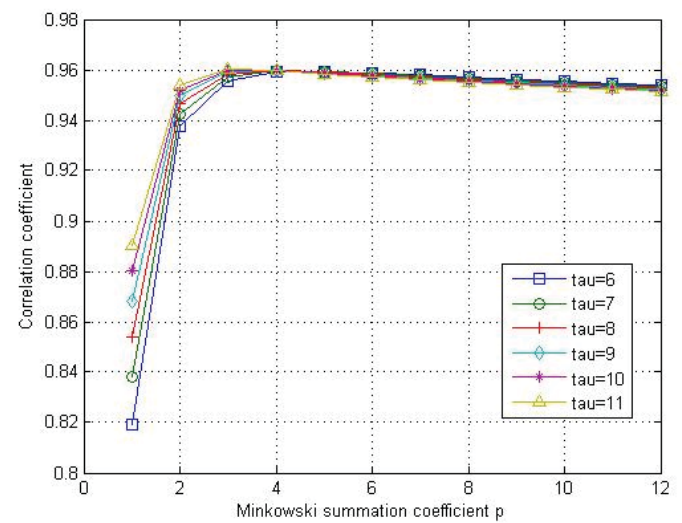

Figure 10: Pearson correlation coefficients for $V_{q}$ with different values of tau and $p$.

There are 6 curves corresponding to 6 different exponential decay rates in total in this figure. The general trend is that higher values of tau corresponds to a better improvement for the correlations when the summation exponent $p$ is in the range 1-3. When $p$ is over 3 , the change of the enhancement for the correlation due to higher tau values is not obvious and even has a very slight decline. Nevertheless, the correlation coefficients are still above 0.95 . On the other hand, when $p$ increases, all curves have the same trend: a sharp increase from 0.82 to 0.96 as $p$ are over the range $1-3$. When $p$ exceeds 3 , all curves have a slight decrease but is still above 0.95 . We could see that all curves have a peak value of the correlation with $p=3$ and all correlation coefficients of different tau values at the peak have little difference. Figure 11 shows the best correlation achieved with exponentially-weighted Minkowski summation.

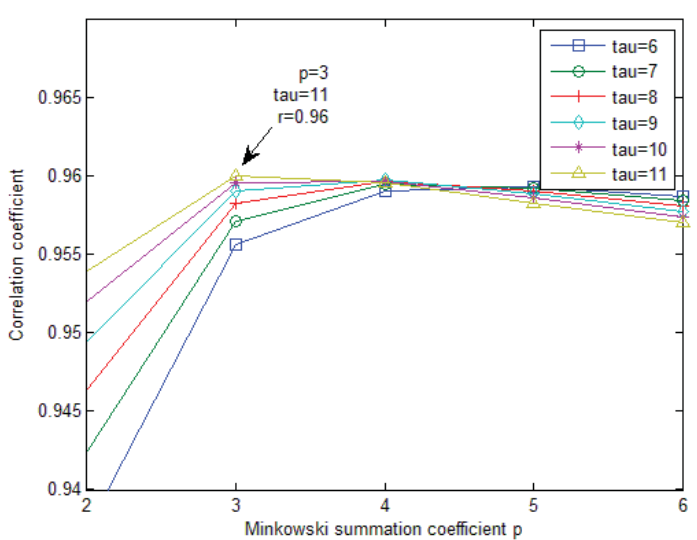

Figure 11: The best correlation coefficients with subjective data.

The correlation results between subjective MOS and the improved model with introducing exponentially-weighted Minkowski summation is shown in Figure 12. Apparently, the improved model gives much better results, achieving a extremely stronger correlation. The correlation coefficients could reach up to 0.96 .

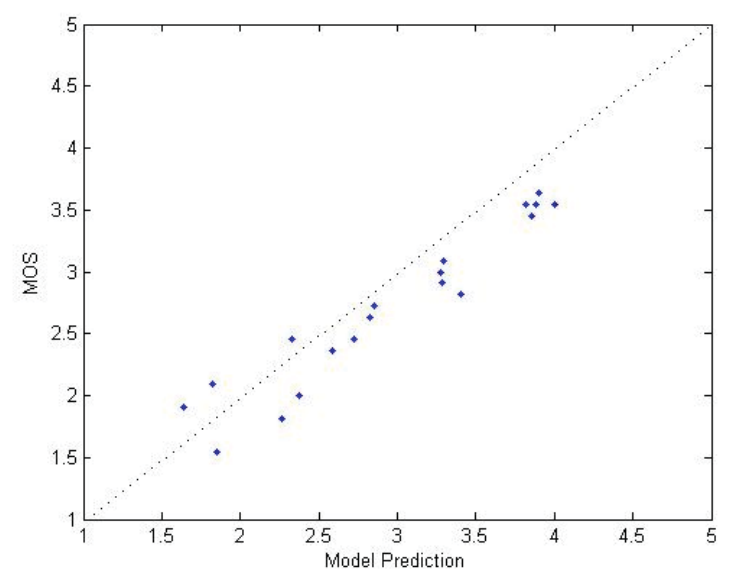

Figure 12: Scatter plots of MOS versus scores calculated from the model with introducing exponentially-weighted Minkowski summation.

Table 7: Performance comparison between the original model and the improved model

\begin{tabular}{lcc}
\hline Models & PCC & RMSE \\
\hline original model & 0.64 & 1.20 \\
improved model & 0.96 & 0.32 \\
\hline
\end{tabular}


Table 7 shows that the objective scores obtained using the model with introducing exponentially-weighted Minkowski summation are much more consistent with subjective MOS.

\section{Conclusion}

Video quality estimation research for LTE video telephony has been motivated by the fact that mobile video services are becoming more and more popular on account of commercial LTE deployment. We present a specific approach to obtain network and media statistic information to analyses the LTE video telephony performance. Additionally, due to a lack of a standard database of test video of video calls over LTE, we have generated a video telephony database which covers a variety of scenarios with different packet loss rates, different bit rates, different movements and in different environments. Subjective tests have been conducted on this database. On the other hand, according to the characteristics of LTE video telephony, we improved the video quality evaluation model based on [8] and [9] by introducing exponentially-weighted Minkowski summation which takes recency effect and peak effect into consideration. The results predicted by this model have a much better correlation with subjective MOS. It could be seen from the previous analysis that when the summation exponent $p=3$, the correlation is the best for different tau values and correlation coefficients could reach up to 0.96 (when tau=11). This means a strong enhancement to the original model without introducing exponentially-weighted Minkowski summation. It was, accordingly, well suited for LTE video telephony applications quality assessment.

\section{ACKNOWLEDGMENTS}

This work was supported by Beijing Natural Science Foundation No.4152047 and 111 Project of China under Grant B14010.

\section{REFERENCES}

[1] Chikkerur, S., Sundaram, V., Reisslein, M. and Karam, L. J., 2011. Objective video quality assessment methods: a classification, review, and performance comparison. IEEE Transactions on Broadcasting, 57,2(2011) 165182.

2] Rohaly, A. M., Lu, J., Franzen, N. R. and Ravel, M. K., 1999. Comparison of temporal pooling methods for estimating the quality of complex video sequences. Proceedings of SPIE - The International Society for Optical Engineering, 3644, 218-225.

[3] GSMA. IR.94 IMS Profile for Conversational Video Service Version 10.0, 2015.

[4] K. Brunnström, K. De Moor, A. Dooms, S. Egger, M.N. Garcia and T. Hossfeld, et al., 2014. Qualinet white paper on definitions of quality of experience. Ntnu.

[5] Video over LTE, https://en.wikipedia.org/wiki/,

[6] Chen, Y., Wu, K., and Zhang, Q., 2015. From qos to qoe: a tutorial on video quality assessment. IEEE Communications Surveys \& Tutorials, 17,2(2015), 1126-1165.

[7] ITU. ITU-T Recommendation G.1070: Opinion model for video-telephony applications. 2007

[8] Joskowicz, J. and Ardao, J. C. L., 2009. Enhancements to the opinion model for video-telephony applications. International Latin American NETWORKING Conference (Vol.87, pp.87-94). ACM.

[9] Yamagishi, K. and Hayashi, T., 2008. Parametric Packet-Layer Model for Monitoring Video Quality of IPTV Services. IEEE International Conference on Communications (Vol.92-A, pp.110-114). IEEE.

[10] Tcpdump, http://www.tcpdump.org

[11] Wireshark, https://www.wireshark.org
12] Pappas, T. N., 1998. Viewer response to time-varying video quality. Proceedings of SPIE - The International Society for Optical Engineering, 3299(1), 16-25.

[13] Yang, F. and Wan, S., 2012. Bitstream-based quality assessment for networked video: a review. IEEE Communications Magazine, 50,11(2012), 203-209.

14] Chikkerur, S., Sundaram, V., Reisslein, M. and Karam, L. J., 2011. Objective video quality assessment methods: a classification, review, and performance comparison. IEEE Transactions on Broadcasting, 57,2(2011), performan-182.

[15] Deng, X., Chen, L., Wang, F. and Fei, Z. 2014. A Novel Strategy to Evaluate QoE for Video Service Delivered over HTTP Adaptive Streaming. IEEE, Vehicular Technology Conference (pp.1-5). IEEE.

[16] Wang, F., Fei, Z., Wang, J., Liu, Y. and Wu, Z., 2017. Has qoe prediction based on dynamic video features with data mining in lte network. Science China Information Sciences, 60,4(2017), 042404

[17] Rimac-Drlje, S., Vranjes, M. and Zagar, D., 2009. Influence of temporal pooling method on the objective video quality evaluation. IEEE International Symposium on Broadband Multimedia Systems and Broadcasting (pp.1-5). IEEE.

[18] Zheng, K., Zhang, X., Zheng, Q., and Xiang, W., 2015. Quality of experience assessment and its application to video services in LTE networks. IEEE Wireless Communications, 22,1(2015), 70-78.

[19] Hermanns, N., Hamm, L., and Sarker, Z., 2014. A Framework and Evaluation of Rate Adaptive Video Telephony in 4G LTE WTC 2014; World Telecommunications Congress 2014; Proceedings of (pp.1-6). VDE.

[20] Wang, F., Chen, L., Deng, X. L., Fei, Z. S., Han, G. L. and Wan, L., 2016. Evaluate mobile video quality with LTE radio access network parameters. fournal of Beijing Institute of Technology, 25,4(2016), 553-561.

[21] Chen, L. Deng X Oi, Y and Mohammed, S. A , 2014. A Method to Evaluate Quality of Real Time Video Streaming in Radio Access Network. IEEE International Conference on Computer and Information Technology (pp.340344). IEEE.

[22] Vaser, M. and Forconi, S., 2015. QoS KPI and QoE KQI Relationship for LTE VIdeo Streaming and VoLTE Services. International Conference on Next Generation Mobile Applications, Services and Technologies (pp.318-323). 